\title{
Nonlinear State Feedback Synthesis by Global Input/Output Linearization
}

This paper studies the design of feedback controllers for trajectory tracking in single-input/single-output nonlinear systems $\dot{x}=f(x)+g(x) u$, $y=h(x)$. A nonlinear transformation of the form $v=\kappa(x)+\lambda(x) u$ that transforms this nonlinear input/output system into a linear system is first constructed. On the basis of this transformation, an approach for designing control laws for trajectory tracking is presented. The control law is robust in the sense that small changes in it do not produce large steady state errors or loss of stability. The theory provides a unified framework for treating control problems arising in nonlinear chemical processes; this is illustrated by a batch reactor control example.

\author{
Costas Kravaris and \\ Chang-Bock Chung \\ Department of Chemical Engineering \\ University of Michigan \\ Ann Arbor, Ml 48109
}

\section{Introduction}

In chemical engineering there is a plethora of nonlinear processes; their nonlinear behavior seems to be well understood especially in the field of chemical reaction engineering, where there is a wide body of literature on steady state multiplicities and nonlinear oscillations in chemical reactors. However, in the process control field the customary approach has been to neglect these nonlinear effects by locally linearizing the nonlinear model around the operating conditions and then to apply linear theory to design linear controllers. For continuous processes with relatively mild nonlinearities that operate around steady states, the error introduced by locally linearizing around the steady state may be small enough so that it can be rejected easily by a sufficiently robust linear regulator. For the regulation of severely nonlinear chemical processes $(\mathrm{pH}$ processes, steam jets, some nonisothermal chemical reactors) a linear controller based on a linear model may have extremely poor performance and the use of nonlinear control elements may be a necessity (Shinskey, 1979). The difficulties in applying linear theory for nonlinear processes are aggravated in servo control problems such as startup/shut-down of continuous processes or optimal profile tracking of batch processes; in these cases there is no appropriate point for local linearization.

Any attempt to avoid the linear approximation step and study general nonlinear systems with general nonlinear control laws will face enormous technical difficulties that will prevent the development of general design methodologies. The only way to bypass these difficulties is to judiciously restrict the class of nonlinear control laws under consideration so that (i) the closed-

Correspondence concerning this paper should be addressed to Costas Kravaris. loop responses can be easily analyzed; (ii) the class of control laws is rich enough to provide the flexibility of choosing one that gives desirable closed-loop characteristics.

In this direction, there has been considerable effort in the recent literature to "globally" linearize nonlinear systems. Global linearization involves finding global nonlinear transformations on the states and/or the manipulated inputs so that the transformed system possesses certain linearity characteristics. The idea was initiated by Brockett (1978) and further advanced by $\mathrm{Su}$, Hunt, and Meyer ( $\mathrm{Su}, 1982$; Hunt et al., 1983), who presented a general method of construction of state variable transformations and a state feedback transformation that transform a nonlinear state model into a linear state model with prespecified dynamic characteristics. Their approach provides a simple and effective method for state feedback stabilization of nonlinear systems. In fact, Hoo and Kantor $(1985,1986)$ have successfully applied it for the state feedback stabilization of unstable chemical and biological reactors. For the very special case of strictly first-order nonlinear systems, Ogunnaike (1986) presented a similar state-variable transformation technique by which special linear PI-controllable systems can be obtained. However, the use of the Su-Hunt-Meyer theory for set point tracking in the presence of disturbances seems to present theoretical difficulties due to the fact that the controlled output will not in general depend linearly on the transformed states.

The idea of globally linearizing a nonlinear system in an input/output sense was first introduced by Gilbert and $\mathrm{Ha}$ (1984). For trajectory tracking in a restricted class of nonlinear systems describing robot manipulators, they considered the problem of finding state-variable transformations and a state feedback control law that provide not only linearity of the trans- 
formed state model but also a linear tracking error equation. The linearity of the error equation is extremely advantageous for design purposes and, once obtained, can be shown to lead to robust controller designs (Ha and Gilbert, 1985). However, in order to find such powerful transformations that globally linearize the state model and the tracking error equation, one will have to make a very restrictive assumption on the process dynamics, namely that the order of the process must be equal to the relative order of the process (see the section on input/output linearization below); this makes the applicability of the Gilbert-Ha work quite limited.

In this work we are going to define and solve a more general input/output linearization problem in which we will only be seeking linearity of the transformed input/output map; the state model need not be transformed into a linear one. At this stage, the analysis is restricted to single-input/single-output (SISO) systems of the form

$$
\begin{aligned}
\dot{x}_{I}= & f_{1}\left(x_{1}, \ldots, x_{n}\right)+g_{1}\left(x_{1}, \ldots, x_{n}\right) u \\
& \vdots \\
\dot{x}_{n} & =f_{n}\left(x_{1}, \ldots, x_{n}\right)+g_{n}\left(x_{1}, \ldots, x_{n}\right) u \\
y & =h\left(x_{1}, \ldots, x_{n}\right)
\end{aligned}
$$

where $u$ is the manipulated input, $x_{1}, \ldots, x_{n}$ are the states, and $y$ is the output. It is noteworthy that almost all SISO chemical processes can be described by a model of the form of Eq. 1, in which the righthand side is a linear function of the manipulated input $u$. For such processes, a nonlinear algebraic transformation $v=\Omega(x, u)$ is sought such that the dependence of $y$ on $v$ is linear. A simple method for construction of such a transformation is presented, such that the order of the transformed input/ output system is minimal. Once the transformation is constructed, a controller design method is presented for trajectory tracking that is robust with respect to small errors in the linearizing transformation. The approach is applied to a temperature tracking problem of a batch reactor. The simulation study shows a good tracking performance of the feedback controller designed as above, despite significant process noise and modeling error.

The approach outlined in this work is a novel general framework for the design of control systems for nonlinear processes. By transforming a nonlinear input/output system into a linear input/output system, the nonlinear process control system design problem reduces to the linear control system design problem. Thus, the heritage of linear control theory is effectively used for the development of powerful control methods for nonlinear systems.

\section{Mathematical Preliminaries}

Given $f$, a $C^{\infty}$ vector field on $R^{n}$, and $h$, a $C^{\infty}$ scalar field on $R^{n}$, the Lie derivative of $h$ with respect to $f$ is defined as

$$
L_{f}(h)=\langle d h, f\rangle
$$

where $\langle\cdot, \cdot\rangle$ denotes the dual product, i.e.,

$$
\langle d h, f\rangle=\frac{\partial h}{\partial x_{1}} f_{1}+\cdots+\frac{\partial h}{\partial x_{n}} f_{n}
$$

This Lie derivative is also a $C^{\infty}$ scalar field on $R^{n}$. Thus, one can inductively define higher order Lie derivatives as follows:

$$
L_{f}^{k}(h)=L_{f}\left[L_{f}^{k-1}(h)\right]=\left\langle d L_{f}^{k-1}(h), f\right\rangle, \quad k=2,3, \ldots
$$

Given $f, g C^{\infty}$ vector fields on $R^{n}$, the Lie bracket $[f, g]$ is a vector field defined by

$$
[f, g]=\frac{\partial g}{\partial x} f-\frac{\partial f}{\partial x} g
$$

where $\partial f / \partial x$ and $\partial g / \partial x$ are the Jacobians. $[f, g]$ is also a $C^{\infty}$ vector field on $R^{n}$, and one can define successive Lie brackets $[f,[f, g]] ;[f,[f,[f, g]]]$; etc. We will use the standard notation

$$
\begin{aligned}
a d_{f}^{0}(g)= & g \\
\operatorname{ad}_{f}^{1}(g)= & {[f, g] } \\
\operatorname{ad}_{f}^{2}(g)= & {[f,[f, g]] } \\
& \vdots \\
\operatorname{ad}_{f}^{k}(g)= & {\left[f, a d_{f}^{k-1}(g)\right] }
\end{aligned}
$$

The following Leibnitz-type formula

$$
\begin{aligned}
\left\langle d L_{f}^{k}(h), g\right\rangle= & L_{f}^{k}(\langle d h, g\rangle)-k L_{f}^{k-1}\left[\left\langle d h, a d_{f}^{1}(g)\right\rangle\right] \\
& +\frac{k(k-1)}{2 !} L_{f}^{k-2}\left[\left\langle d h, a d_{f}^{2}(g)\right\rangle\right] \\
& -\frac{k(k-1)(k-2)}{3 !} L_{f}^{k-3}\left[\left\langle d h, a d_{f}^{3}(g)\right\rangle\right] \\
& + \\
& \vdots \\
& + \\
& +(-1)^{k}\left\langle d h, a d_{f}^{k}(g)\right\rangle, \quad k=1,2, \ldots
\end{aligned}
$$

will be useful.

\section{Su-Hunt-Meyer Linearization}

Su, Hunt, and Meyer (Su, 1982; Hunt et al., 1983) studied the problem of transforming a nonlinear system of the form

$$
\dot{x}=f(x)+g(x) u
$$

where $f$ and $g$ are $C^{\infty}$ vector fields on $R^{n}$ and $u \in R$, into a controllable linear system of the form

$$
\dot{\xi}=A \xi+b v
$$

where $A$ and $b$ are $n \times n$ and $n \times 1$ matrices, respectively, through transformations of the form

$$
\xi_{1}=\Xi_{1}(x)
$$

$$
\begin{aligned}
\xi_{n} & =\Xi_{n}(x) \\
v & =\Xi_{n+1}(x, u), \quad \text { with } \frac{\partial \Xi_{n+1}}{\partial u} \neq 0
\end{aligned}
$$


Since every controllable linear system of the form of Eq. 7 is equivalent to a string of integrators

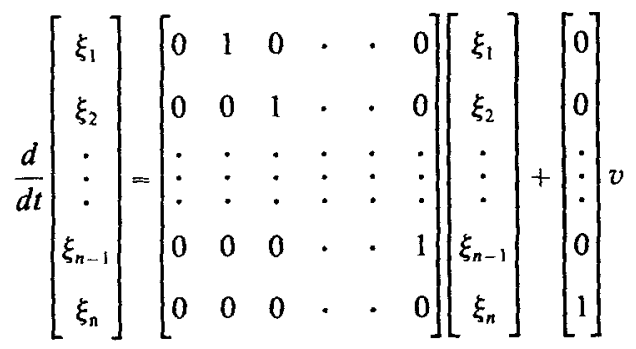

their problem reduced to that of finding transformations as in Eq. 8 that transform Eq. 6 into Eq. 9. They showed that a necessary and sufficient condition for the existence of such transformations is the existence of a scalar field $q(x)$ satisfying

$$
\left\{\begin{array}{l}
\left\langle d q, a d_{f}^{i}(g)\right\rangle=0, \quad i=0,1, \ldots, n-2 \\
\left\langle d q, a d_{f}^{n-1}(g)\right\rangle \neq 0
\end{array}\right.
$$

Using the well-known theorem of Frobenius (Boothby, 1975) they concluded that the latter condition is equivalent to

$$
\left\{\begin{array}{l}
g, a d_{f}^{1}(g), \ldots, a d_{f}^{n-1}(g) \text { are linearly independent } \\
\text { The set of vector fields }\left\{g, a d_{f}^{1}(g), \ldots, a d_{f}^{n-2}(g)\right\} \\
\text { is involutive. }
\end{array}\right.
$$

Once a $q$ satisfying Eq. 10 is specified, the set of transformations in Eq. 8 is constructed in a straightforward fashion:

$$
\begin{aligned}
& \Xi_{1}=q \\
& \Xi_{2}=\left\langle d \Xi_{1}, f\right\rangle \\
& \Xi_{3}=\left\langle d \Xi_{2}, f\right\rangle \\
& \vdots \\
& \vdots \\
& \Xi_{n}=\left\langle d \Xi_{n-1}, f\right\rangle \\
& \Xi_{n+1}=\left\langle d \Xi_{n}, f\right\rangle+\left\langle d \Xi_{n}, g\right\rangle_{u}
\end{aligned}
$$

The Su-Hunt-Meyer approach provides a very general method of transforming a nonlinear state model into a linear state model. Notice, however, that in process control problems the objective is usually to force an output $y=h\left(x_{1}, x_{2}, \ldots, x_{n}\right)$ to follow a set point in the presence of disturbances. This output will not in general depend linearly on the transformed states $\xi_{1}$, $\xi_{2}, \ldots, \xi_{n}$ since the composite $\operatorname{map} h\left[\Xi_{1}(\cdot), \Xi_{2}(\cdot), \ldots, \Xi_{n}(\cdot)\right]$ will not in general be linear. The (in general) nonlinearity of the $v-y$ system is a major disadvantage of the Su-Hunt-Meyer approach. To overcome this disadvantage, Kantor (1986) suggested that the control engineer could in some cases redefine the output so that it depends linearly on the transformed states $\xi_{1}$, $\ldots, \xi$. Unless a physically meaningful output that depends linearly on the $\xi$ 's can be found, there will be serious difficulties in the design of control laws.

The Su-Hunt-Meyer approach has other disadvantages, namely:

1. The involutivity condition is rather restrictive. For exam- ple, systems with $g(x)$ having only one nonzero component will in general fail to meet the involutivity requirement. Such systems can be, for example, nonisothermal chemical reactors whose temperature is controlled by manipulating the heat inflow (see the final section below).

2. Even if the involutivity condition is met and therefore a transformation $\Xi$ exists, its calculation is not at all trivial. One will first have to solve the partial differential equations for $q$ arising from Eq. 10; these may not admit a closed-form solution and their numerical solution can be quite involved.

\section{Input/Output Linearization}

Consider an input/output system of the form

$$
\begin{aligned}
& \dot{x}=f(x)+g(x) u \\
& y=h(x)
\end{aligned}
$$

where $f$ and $g$ are $C^{\infty}$ vector fields in $R^{n}, h$ is a $C^{\infty}$ scalar field on $R^{n}$, and $u \in R$.

Definition 1. A system of the form of Eq. 13 is called input/ output linearizable if there exists a $C^{\infty}$ function from $R^{n+1}$ to $R^{n}$

$$
v=\Omega(x, u)
$$

with $\partial \Omega / \partial u \neq 0$ and a linear differential operator of the form

$$
\mathcal{L}_{r}=\sum_{k=0}^{r} \beta_{k} \frac{d^{k}}{d t^{k}}
$$

such that

$$
\mathcal{L}_{r} y=v
$$

Definition 2. Assume that Eq. 13 is input/output linearizable. The smallest integer $r$ for which there exists a transformation $v=\Omega(x, u)$ and a differential operator $\mathcal{L}$, of the form of Eq. 15 so that Eq. 16 is satisfied, is called the linearizability index of Eq. 13.

Theorem 1. A necessary and sufficient condition for input/ output linearizability is the existence of a positive integer $r$ such that

$$
\left\langle d h, a d_{f}^{r-1}(g)\right\rangle \neq 0
$$

The linearizability index is the smallest integer $r$ for which $E q$. 17 is satisfied.

Proof. We will first show sufficiency. Without loss of generality, assume that

$$
\begin{gathered}
\left\langle d h, a d_{f}^{k}(g)\right\rangle=0 \quad k=0,1, \ldots, r-2 \\
\left\langle d h, a d_{f}^{r-1}(g)\right\rangle \neq 0
\end{gathered}
$$

From Eq. 5 it easily follows that

$$
\begin{aligned}
\left\langle d L_{f}^{k}(h), g\right\rangle & =0, \quad k=1, \ldots, r-2 \\
\left\langle d L_{f}^{r-1}(h), g\right\rangle & =(-1)^{r-1}\left\langle d h, a d_{f}^{r-1}(g)\right\rangle
\end{aligned}
$$


Thus

$$
\begin{aligned}
& \frac{d^{k} y}{d t^{k}}=L_{f}^{k}(h), \quad k=1, \ldots, r-1 \\
& \frac{d^{\prime} y}{d t^{r}}=L_{f}^{r}(h)+(-1)^{r-1}\left\langle d h, a d_{f}^{r-1}(g)\right\rangle u
\end{aligned}
$$

It follows immediately that the transformation

$$
v=\Omega(x, u)=L_{f}^{r}(h)+(-1)^{r-1}\left\langle d h, a d_{f}^{r-1}(g)\right\rangle u
$$

satisfies $\partial \Omega / \partial u \neq 0$ and $d^{r} y / d t^{r}=v$, i.e., linearizes the input/ output system, Eq. 13.

To show necessity, assume that

$$
\left\langle d h, a d_{f}^{k-1}(g)\right\rangle=0 \quad \text { for every } k \in N
$$

Then from Eq. 5 one easily obtains by induction that

$$
\left\langle d L_{f}^{k-1}(h), g\right\rangle=0 \quad \text { for every } k \in N
$$

But then

$$
\frac{d^{k} y}{d t^{k}}=L_{f}^{k}(h) \quad \text { for every } k \in N
$$

and so

$$
\mathcal{L}_{r} y=\sum_{k=0}^{r} \beta_{\mathrm{k}} \frac{d^{k} y}{d t^{k}}=\sum_{k=0}^{r} \beta_{k} L_{f}^{k}(h)
$$

is independent of $u$ for every $r$ and every set $\left\{\beta_{k}\right\}$. Therefore, all the transformations $v=\Omega(x, u)$ that satisfy an equation of the form of Eq. 16 will have $\partial \Omega / \partial u=0$. So the system will not be input/output linearizable.

To complete the proof, let $r$ be the linearizability index of Eq. 13 and assume that there exists $r^{\prime}<r$ such that $\left\langle d h, a d_{f}^{r^{\prime}-1}(g)\right\rangle$ $\neq 0$. Then the transformation

$$
v=\Omega(x, u)=L_{f}^{r^{\prime}}(h)+(-1)^{r^{\prime}-1}\left\langle d h, a d_{f}^{r^{\prime}-1}(g)\right\rangle u
$$

will satisfy $\partial \Omega / \partial u \neq 0$ and $d^{r^{\prime}} y / d t^{r^{\prime}}=v$. This leads to contradiction.

Remark 1. It is interesting to note that in the special case of a linear system $\dot{x}=A x+b u, y=c x$, i.e., when $f(x)=A x, g(x)=$ $b, h(x)=c x$, we have

$$
(-1)^{i}\left\langle d h, \operatorname{ad}_{f}^{i}(g)\right\rangle=c A^{i} b, \quad i=0,1,2, \ldots
$$

In other words, $(-1)^{i}\left\langle d h, a d_{f}^{i}(g)\right\rangle, i=0,1,2, \ldots$, are the Markov parameters of the input/output system. Then the smallest integer $r$ for which Eq. 17 is satisfied is simply the relative order of the input/output system. In nonlinear system theory, the relative order is defined as the smallest integer for which Eq. 17 is satisfied (Hirschorn 1979). Using this definition one has the following:

Corollary 1. A system is input/output linearizable if and only if it has finite relative order. The linearizability index is equal to the relative order of the system.
Remark 2. In the work of Hirschorn (1979) it is proved that a nonlinear system is left-invertible if and only if it has finite relative order. Thus, we have the following corollary:

Corollary 2. A sytem is input/output linearizable if and only if it is left-invertible.

Theorem 2. Assume that Eq. 13 is input/output linearizable with linearizability index $r$. Then the state feedback transformation

$$
v=\Omega(x, u)=\sum_{k=0}^{r} \beta_{k} L_{f}^{k}(h)+(-1)^{r-1} \beta_{r}\left\langle d h, a d_{f}^{r-1}(g)\right\rangle u
$$

transforms the input/output system of Eq. 13 into

$$
\sum_{k=0}^{r} \beta_{k} \frac{d^{k} y}{d t^{k}}=v
$$

Proof. Immediate consequence of Eq. 18.

Remark 3. When the linearizability index $r=n$, i.e.,

$$
\begin{gathered}
\left\langle d h, a d_{f}^{k}(g)\right\rangle=0, \quad k=1, \ldots, n-1 \\
\left\langle d h, a d_{f}^{n-1}(g)\right\rangle \neq 0
\end{gathered}
$$

then $q=h$ satisfies Eq. 10 . Therefore, by choosing according to Eq. 12

$$
\begin{gathered}
\xi_{1}=\Xi_{1}(x)=h(x) \\
\xi_{2}=\Xi_{2}(x)=\left\langle d \Xi_{1}, f\right\rangle=\langle d h, f\rangle=L_{f}(h) \\
\xi_{3}=\Xi_{3}(x)=\left\langle d \Xi_{2}, f\right\rangle=\left\langle d L_{f}(h), f\right\rangle=L_{f}^{2}(h) \\
\vdots \\
\vdots \\
\xi_{n}=\Xi_{n}(x)=\left\langle d \Xi_{n-1}, f\right\rangle=\left\langle d L_{f}^{n-2}(h), f\right\rangle=L_{f}^{n-1}(h) \\
\Xi_{n+1}(x, u)=\left\langle d \Xi_{n}, f\right\rangle+\left\langle d \Xi_{n}, g\right\rangle u \\
=\left\langle d L_{f}^{n-1}(h), f\right\rangle+\left\langle d L_{f}^{n-1}(h), g\right\rangle u \\
=L_{f}^{n}(h)+(-1)^{n-1}\left\langle d h, a d_{f}^{n-1}(g)\right\rangle u
\end{gathered}
$$

Eq. 13 is transformed into Eq. 9. But then $d^{n} y / d t^{n}=v$, i.e., the system is also linearized in an input/output sense. So, when $r=$ $n$ :

1. The Su-Hunt-Meyer linearization can provide an input/ output linearization as well.

2. The input/output linearizing transformation

$$
v=\Omega(x, u)=L_{f}^{n}(h)+(-1)^{n-1}\left\langle d h, a d_{f}^{n-1}(g)\right\rangle u
$$

in conjunction with the state variable transformation

$$
\xi_{i}=L_{f}^{i-1}(h), \quad i=1, \ldots, n
$$

linearizes the state equations as well.

Thus the Su-Hunt-Meyer linearization can be viewed as a special case of input/output linearization when the linearizability index is equal to the order of the system; under this restrictive condition, both approaches solve the problem of Gilbert and $\mathrm{Ha}$.

Remark 4. The state feedback transformation, Eq. 19, transforms the input/output system of Eq. 13 into a linear system of 
minimal order. This follows immediately from the definition of the linearizability index $r$. It may be possible to find state feedback transformations $v=\Omega(x, u)$ that transform Eq. 13 into linear systems of higher order.

Remark 5. The assumption of input/output linearizability is a very weak one. If a system is not input/output linearizable, then $y$ and its derivatives of all orders are independent of $u$. Such uncontrollable systems are of no practical interest.

Remark 6 . There may be singular lines in the phase space for which $\partial \Omega / \partial u=\beta_{r}(-1)^{r-1}\left\langle d h, a d_{f}^{r-1}(g)\right\rangle=0$. These can be viewed as lines of linearizability index higher than $r$. Consideration of these singularities is beyond the scope of this work.

\section{Globally Linearizing Controllers}

We saw in the previous section that under the state feedback transformation, Eq. 19, or equivalently

$$
u=\Psi(x, v)=\frac{v-\sum_{k-0}^{r} \beta_{k} L_{f}^{k}(h)}{(-1)^{r-1} \beta_{r}\left\langle d h, a d_{f}^{r-1}(g)\right\rangle}
$$

an input/output system of the form of Eq. 13 with linearizability index $r$ transforms into Eq. 20. Note that the parameters $\beta_{0}, \beta_{1}$, $\ldots, \beta$, are completely arbitrary, which means that the $v-y$ system can have arbitrarily placed poles and, therefore, prespecified bounded-input/bounded-output (BIBO) stability characteristics. When the poles of the $v-y$ system are appropriately placed "far left" in the complex plane, one can use an external PI loop

$$
v=K_{c}\left[\left(y_{\mathrm{d}}-y\right)+\frac{1}{\tau_{I}} \int_{0}^{t}\left(y_{\mathrm{d}}-y\right)\right]
$$

to force the output $y(t)$ to track a given desired trajectory $y_{\mathrm{d}}(t)$. The resulting control structure, which we call the globally linearizing control (GLC) structure is depicted in Figure 1. Combining Eqs. 20 and 22, we obtain the closed-loop response of the GLC structure. This is the solution of

$$
\sum_{k=0}^{r} \beta_{k} \frac{d^{k} y}{d t^{k}}=K_{c}\left[\left(y_{\mathrm{d}}-y\right)+\frac{1}{\tau_{I}} \int_{0}^{t}\left(y_{\mathrm{d}}-y\right)\right]
$$

which can be computed by standard Laplace transform techniques. It follows that the closed-loop system will be BIBO stable and the tracking error $y_{d}(t)-y(t)$ will eventually go to zero if and only if the roots of the characteristic equation

$$
\beta_{r} s^{r}+\beta_{r-1} s^{r-1}+\cdots+\beta_{1} s+\left(\beta_{0}+K_{c}\right)+\frac{K_{c}}{\tau_{f} s}=0
$$

have negative real parts.

It is important to note that the GLC structure is equivalent to standard nonlinear feedback with control law

$$
u=\frac{K_{c}\left[\left(y_{\mathrm{d}}-y\right)+\frac{1}{\tau_{I}} \int_{0}^{1}\left(y_{\mathrm{d}}-y\right)\right]-\sum_{k=0}^{r} \beta_{k} L_{f}^{k}(h)}{(-1)^{r-1} \beta_{r}\left(d h, a d_{f}^{r-1}(g)\right\rangle}
$$

This follows readily from Eqs. 21 and 22.

The design procedure using the GLC structure is extremely simple and can be summarized as follows:

1. Compute the linearizing transformation, Eq. 19, from the system model.

2. Select $\left\{\beta_{k}\right\}$ so that the poles of Eq. 20 are far left in the complex plane.

3. Tune a PI controller for Eq. 20.

Remark 7. More generally, one can use a non-PI linear controller, which is appropriately tuned for Eq. 20.

\section{Robustness Analysis}

We saw in the previous section that the nonlinear control law

$$
u=\frac{K_{c}\left[\left(y_{\mathrm{d}}-y\right)+\frac{1}{\tau_{1}} \int_{0}^{1}\left(y_{\mathrm{d}}-y\right)\right]-\kappa(x)}{\lambda(x)}
$$

with

$$
\begin{aligned}
& \kappa(x)=\sum_{k=0}^{r} \beta_{k} L_{f}^{k}(h), \\
& \lambda(x)=(-1)^{r-1} \beta_{r}\left\langle d h, a d_{f}^{r-1}(g)\right\rangle
\end{aligned}
$$

provides a prespecified closed-loop response, Eq. 23, for a system of the form of Eq. 13. The closed-loop error $y_{d}-y$ goes asymptotically to zero with speed determined by the roots of the characteristic equation, Eq. 24.

In practice, due to modeling errors $\kappa(x)$ and $\lambda(x)$ will be calculated inaccurately, and therefore the actual closed-loop response will deviate from the prespecified one, Eq. 23. Thus the question is whether a control law of the form of Eq. 26 with inac-

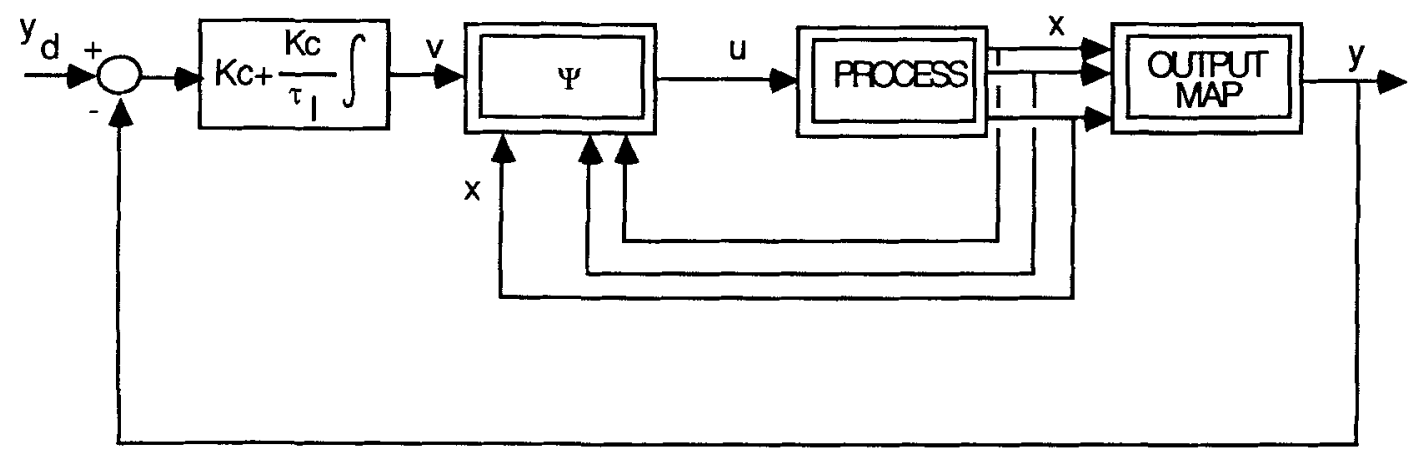

Figure 1. Globally linearizing control (GLC) structure. 
curate $\kappa$ and $\lambda$ will be able to approximately bring the closedloop error $y_{\mathrm{d}}(t)-y(t)$ to zero in a stable fashion.

Let $v=\kappa(x)+\lambda(x) u$ be an exact linearizing transformation of Eq. 13, and $\bar{v}=\bar{\kappa}(x)+\bar{\lambda}(x) u$ an approximately linearizing transformation, where $\kappa, \bar{\kappa}, \lambda, \bar{\lambda}$ are all $C^{\infty}$ vector fields on $R^{n}$. Consider

$$
\frac{d}{d t}\left[\begin{array}{l}
x \\
z
\end{array}\right]=\left[\begin{array}{c}
f(x)+g(x) \frac{K_{c}\left\{\left[y_{\mathrm{d}}-h(x)\right]+\frac{z}{\tau_{l}}\right\}-k(x)}{\lambda(x)} \\
y_{\mathrm{d}}-h(x)
\end{array}\right]
$$

and

$$
\frac{d}{d t}\left[\begin{array}{l}
\bar{x} \\
\bar{z}
\end{array}\right]=\left[\begin{array}{c}
\left.f(\bar{x})+g(\bar{x}) \frac{K_{c}\left\{\left[y_{\mathrm{d}}-h(\bar{x})\right]+\frac{\bar{z}}{\tau_{l}}\right\}-\bar{\kappa}(\bar{x})}{\bar{\lambda}(\bar{x})}\right] \\
y_{\mathrm{d}}-h(\bar{x})
\end{array}\right]
$$

Equation 27 represents the closed-loop system with an exactly linearizing control law, whereas Eq. 28 represents the closedloop system with an approximately linearizing control law.

To simplify the analysis, it will be assumed that $y_{\mathrm{d}}$ is a constant. Perturbation theory for nonlinear systems (Hirsch and Smale, 1974) will be used to study the accuracy of the equilibrium solution and its asymptotic stability. The same perturbation theory was used previously in a similar context ( $\mathrm{Su}$ et al., 1983; Gilbert and $\mathrm{Ha}, 1984)$.

First, it is necessary to introduce appropriate measures of functional closeness and other notation. For an open set $W \subset R^{n}$, let $C^{1}(W)$ be the set of all $C^{1}$ vector fields on $W$. Let $|\cdot|$ be the usual norm for $R^{n}$ and $\|\cdot\|$ be the usual operator norm in the set of $n \times n$ matrices. Then the $C^{l}$ norm $\|f\|_{1}$ of a vector field $f \in C^{\prime}(W)$ is defined as

$$
\|f\|_{i}=\sup _{x \in W}\left\{|f(x)|,\left\|\frac{\partial f}{\partial x}(x)\right\|\right\}
$$

where $(\partial f / \partial x)(x)$ is the Jacobian matrix of $f$. Thus, two vector fields $f$ and $\bar{f}$ are " $C$ close" if and only if the functions and their derivatives are close. It is also necessary to define neighborhoods in $W$ and $C^{1}(W)$. Given $x^{*} \in W$ and $\epsilon>0$, define

$$
N_{\varepsilon}\left(x^{*}\right)=\left\{x \in W:\left|x-x^{*}\right|<\epsilon\right\}
$$

Given $f \in C^{1}(W)$ and $\eta>0$ define

$$
N_{\eta}(f)=\left\{\bar{f} \in C^{1}(W):\|\bar{f}-f\|_{1}<\eta\right\}
$$

Theorem 3. Let $\left(x^{*}, z^{*}\right)$ be an asymptotically stable equilibrium point of Eq. 27 satisfying $h\left(x^{*}\right)=y_{\mathrm{d}}$. Then for any $\epsilon>0$ there exist $\delta_{k}>0$ and $\delta_{\lambda}>0$ such that for $\|\bar{\kappa}-\kappa\|_{1}<\delta_{k}$ and $\|\bar{\lambda}-\lambda\|_{1}<\delta_{\lambda}$, Eq. 28 admits a unique equilibrium solution $(\bar{x}, \bar{z})$ that is asymptotically stable and satisfres $\left|y_{\mathrm{d}}-h(\bar{x})\right|<\epsilon$.

Remark 8 . The only assumption in Theorem 3 is the existence of an asymptotically stable equilibrium $\left(x^{*}, z^{*}\right)$ of Eq. 27 . That $h\left(x^{*}\right)=y_{\mathrm{d}}$ follows immediately from Eq. 27 .
Remark 9. The theorem shows that both the accuracy of the equilibrium solution and asymptotic stability can be maintained if $\bar{\kappa}-\kappa$ and $\bar{\lambda}-\lambda$ are sufficiently small in the indicated way. In practice, $\delta_{k}, \delta_{\lambda}$, and the norms are difficult to evaluate, so the theorem should be viewed as a qualitative rather than a quantitative result.

Remark 10 . The theorem can easily be generalized to include errors in the measurement of $y$.

The proof of Theorem 3 is straightforward given the following result, which is a simple rewording of results formed in Chapter 16 of Hirsch and Smale (1974) (see also Gilbert and Ha, 1984). Details are omitted for brevity.

Theorem 4 (Hirsch and Smale). Suppose $W \subset R^{n}$ is an open set and $F \in C^{1}(W)$. For $x^{*} \in W$ assume $F\left(x^{*}\right)=0$ and the Jacobian $(\partial F / \partial x)\left(x^{*}\right)$ is nonsingular. Then for any $\epsilon>0$ satisfying $N_{\epsilon}\left(x^{*}\right) \subset W$, there exists $\eta>0$ such that for all $\bar{F} \in N_{\eta}(F)$, $\bar{F}(x)=0$ has a unique solution $x=\bar{x}$ satisfying $\left|\bar{x}-x^{*}\right|<\epsilon$. Furthermore, if the real parts of the eigenvalues of $(\partial F / \partial x)\left(x^{*}\right)$ are smaller than $\sigma<0$, it is possible to choose $\eta$ so that the real parts of the eigenvalues of $(\partial \bar{F} / \partial x)(\bar{x})$ are smaller than $\sigma$.

\section{State Observers}

In the foregoing discussions it is assumed that all the state variables required for the linearizing transformation are available by direct measurement. In practice, however, on-line measurement of all the states is often not possible. In such cases it is necessary to estimate the unavailable states on the basis of a dynamic model and output measurement. Figure 2 shows the globally linearizing control (GLC) structure with a state observer.

For linear systems, the observer theory has been well established and powerful design methods for observers are now widely available (Luenberger, 1966; Kailath, 1980). However, for nonlinear systems, due to their distinct behavior, few methods are available with sufficient generality.

\section{Open-loop observers}

An open-loop observer computes state estimates $\hat{x}(t)$ by solving

$$
\dot{\hat{x}}=f(\hat{x})+g(\hat{x}) u, \quad \hat{x}(0)=\hat{x}_{0}
$$

given on-line measurements of $u$ and estimates of the initial states. For systems with asymptotically stable open-loop dynamics, the open-loop observer given by Eq. 32 provides a simple method of reconstructing the states.

The order of the observer (the number of the states to be estimated) may be reduced by one by making use of the measured output. Specifically, assume that one of the states, say $x_{k}$, can be computed from the state /output relation $y=h(x)$ as follows:

$$
x_{k}=H\left(y, x_{1}, \ldots, x_{k-1}, x_{k+1}, \ldots, x_{n}\right)
$$

Then, a reduced-order open-loop observer is constructed as follows:

$$
\begin{aligned}
\dot{\hat{x}}_{m}= & f_{m}\left(\hat{x}_{1}, \ldots, \hat{x}_{k-1}, H, \hat{x}_{k+1}, \ldots, \hat{x}_{n}\right) \\
& +g_{m}\left(\hat{x}_{1}, \ldots, \hat{x}_{k-1}, H, \hat{x}_{k+1}, \ldots, \hat{x}_{n}\right) u \\
\hat{x}_{m}(0)= & \hat{x}_{m, 0} \\
m= & 1, \ldots, k-1, k+1, \ldots, n .
\end{aligned}
$$




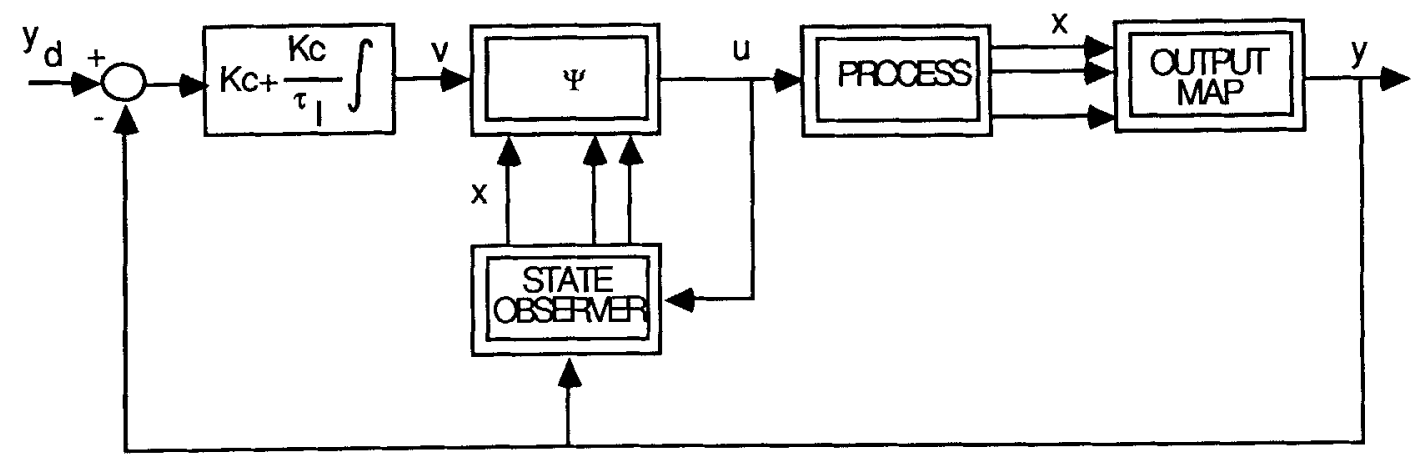

Figure 2. Globally linearizing control structure with a state observer.

\section{Closed-loop observers}

For open-loop unstable processes (where the error of an openloop observer may grow without bound) or open-loop stable processes with slow dynamics (where a significant initial state error may decay quite slowly), a closed-loop observer can be constructed by feeding back the difference between the actual process output and the estimated output as follows:

$$
\dot{\hat{x}}=f(\hat{x})+g(\hat{x}) u+l[y-h(\hat{x})]
$$

where $l$ is an observer gain vector. Determination of the observer gain that provides a fast convergent estimate is a nontrivial task. For systems that operate around a steady state, a popular approach consists of linearizing Eq. 35 around the steady operating point and applying well-known linear observer design methods (Safonov, 1980; Wallman, 1979). Convergence conditions are obtained by using Lyapunov theory, but general and simple conditions are not available. Furthermore, it is very difficult in practice to determine the region around the steady operating point in which the conditions are satisfied. Work on closed-loop nonlinear observers that is not based on linearization around a steady state is restricted to very limited classes of systems (Kou et al., 1975; Banks, 1981; Krener and Isidori, 1983; Bestle and Zeitz, 1983).

The design of convergent closed-loop observers for general nonlinear systems of the form of Eq. 13 is an open problem. In the application to be discussed in the next section, a simple reduced-order open-loop observer of the form of Eq. 34 will be used.

\section{Application to Batch Reactor Control}

Batch processes require a control strategy different from that for continuous processes since they do not operate on steady states. The control objectives is to force the system output to track a desired trajectory.

A common approach to batch reactor control has been to apply optimal control theory in order to obtain optimal state trajectories and/or optimal control laws; these control laws were implemented in an open-loop fashion. A number of papers along this direction are reviewed by Rippin (1983). The common difficulty in these open-loop control strategies was that no compensation was made for modeling errors and random disturbances arising in process operation. As a result, the control strategies were quite sensitive to small errors or disturbances and the subsequent implementations on actual processes were often unsuccessful (Foss, 1973).
The subsequent interest in batch process control has shifted to the design of efficient controller configurations that will make the systems follow an optimal trajectory, which is calculated offline. Several configurations that have been studied include cascade controllers (Marroquin and Luyben, 1972), combined feedback/feedforward controllers (Jutan and Uppal, 1984) and adaptive controllers (Cluett et al., 1985).

In this section we propose the use of globally linearizing controllers (GLC) as an efficient feedback control approach for a class of batch process control problems. As an illustration, we consider a temperature tracking problem of a batch reactor in which the following consecutive reactions take place:

$$
A \stackrel{k_{1}}{\longrightarrow} B \stackrel{k_{2}}{\longrightarrow} C
$$

It is assumed that $A \rightarrow B$ has second-order kinetics whereas $B \rightarrow C$ has first-order kinetics. The component mass balances are then

$$
\begin{gathered}
\frac{d C_{A}}{d t}=-k_{1}(T) C_{A}^{2}, C_{A}(0)=C_{A 0} \\
\frac{d C_{B}}{d t}=k_{1}(T) C_{A}^{2}-k_{2}(T) C_{B}, \quad C_{B}(0)=0
\end{gathered}
$$

where

$$
\begin{aligned}
& k_{1}(T)=A_{10} \exp \left(-E_{1} / \mathrm{R} T\right) \\
& k_{2}(T)=A_{20} \exp \left(-E_{2} / \mathrm{R} T\right)
\end{aligned}
$$

Ray and Szekely (1973) found an optimal temperature trajectory for a maximum yield of $B$ using optimal control theory under the following conditions:

$$
\begin{aligned}
C_{A_{0}} & =1 \\
A_{10} & =1.1 \quad A_{20}=172.2 \\
E_{1} & =2.09 \times 10^{4} \quad E_{2}=4.18 \times 10^{4} \\
T_{\min } & =25 \quad T_{\text {max }}=125
\end{aligned}
$$

Batch time $=1 \mathrm{~h}$.

The control objective in this example is to make the reactor track the optimum profile as closely as possible using GLC synthesis. 
For general servo control of temperature, both heating and cooling of the process unit is necessary. For this batch reactor, the desired heating rate is controlled by regulating the pressure of saturated steam supplied into a heating jacket and, hence, the temperature in the jacket. The desired cooling rate is obtained by manipulating the overall heat transfer coefficient of a cooling coil by adjusting the coolant flow rate. Then the heat balance is given by

$$
\begin{aligned}
\rho C_{p} V \frac{d T}{d t}=k_{1}(T) C_{A}^{2}( & \left.-\Delta H_{1}\right) V+k_{2}(T) C_{B}\left(-\Delta H_{2}\right) V \\
& +U_{j} A_{j}\left(T_{s}-T\right)-U_{c} A_{c}\left(T-T_{c}\right)
\end{aligned}
$$

When two manipulated variables $\left(T_{s}\right.$ and $\left.U_{c}\right)$ are used to control a single output $(T)$, the control system will be over-determined. A way of solving this difficulty is to introduce a single parametric variable $u$, defined in the following manner (Jutan and Uppal 1984):

$$
\begin{aligned}
& T_{s}=\left(T_{s, \text { max }}-T_{s, \text { min }}\right) u+T_{s, \text { min }} \\
& U_{c}=\left(U_{c, \text { min }}-U_{c, \text { max }}\right) u+U_{c, \text { max }}
\end{aligned}
$$

where the maximum and minimum values of $T_{s}$ and $U_{c}$ are chosen from process or safety limits. Clearly, $u=0$ represents the maximum cooling of the system and $u=1$, maximum heating. This parametric variable is used as a single control variable for our tracking problem. After substitution of Eqs. 39 and 40 into Eq. 38 and rearrangement, we obtain

$$
\begin{aligned}
\frac{d T}{d t}=\gamma_{1} k_{1}(T) C_{A}^{2}+\gamma_{2} k_{2}( & T) C_{B} \\
& +\left(a_{1}+a_{2} T\right)+\left(b_{1}+b_{2} T\right) u
\end{aligned}
$$

where

$$
\begin{aligned}
& \gamma_{1}=\left(-\Delta H_{1}\right) / \rho C_{p} \\
& \gamma_{2}=\left(-\Delta H_{2}\right) / \rho C_{p} \\
& a_{1}=\left(U_{j} A_{j} T_{s, \text { min }}+U_{c, \text { max }} A_{c} T_{c}\right) / \rho C_{p} V \\
& a_{2}=-\left(U_{j} A_{j}+U_{c, \text {max}} A_{c}\right) / \rho C_{p} V \\
& b_{1}=\left[U_{j} A_{j}\left(T_{s, \text { max }}-T_{s, \text { min }}\right)-\left(U_{c, \text { max }}-U_{c, \text { min }}\right) A_{c} T_{c}\right] / \rho C_{p} V \\
& b_{2}=\left(U_{c, \text { max }}-U_{c, \text { min }}\right) A_{c} / \rho C_{p} V
\end{aligned}
$$

When we represent the process dynamics, Eqs. 36, 37, and 41, in state variable form, Eq. 13 with $x=\left(C_{A}, C_{B}, T\right)$ and $y=T$, we have

$$
\begin{gathered}
f(x)=\left[\begin{array}{l}
-k_{1}(T) C_{A}^{2} \\
k_{1}(T) C_{A}^{2}-k_{2}(T) C_{B} \\
\gamma_{1} k_{1}(T) C_{A}^{2}+\gamma_{2} k_{2}(T) C_{B}+\left(a_{1}+a_{2} T\right)
\end{array}\right] \\
g(x)=\left[\begin{array}{l}
0 \\
0 \\
b_{1}+b_{2} T
\end{array}\right]
\end{gathered}
$$

$$
h(x)=T
$$

Since

$$
\langle d h, g\rangle=b_{1}+b_{2} T \neq 0,
$$

this nonlinear system is input/output linearizable with the linearizability index $r=1$. A straightforward computation gives

$$
L_{f}(h)=\gamma_{1} k_{1}(T) C_{A}^{2}+\gamma_{2} k_{2}(T) C_{B}+\left(a_{1}+a_{2} T\right)
$$

Now following the design procedure developed previously, each component of GLC is easily obtained as follows:

\section{Transformation}

$$
\begin{aligned}
v=\beta_{0} T+\beta_{1}\left[\gamma_{1} k_{1}(T) C_{A}^{2}+\gamma_{2}\right. & k_{2}(T) C_{B} \\
& \left.+\left(a_{1}+a_{2} T\right)\right]+\beta_{1}\left(b_{1}+b_{2} T\right) u
\end{aligned}
$$

or equivalently

$$
\begin{aligned}
u & =\Psi\left(C_{A}, C_{B}, T, v\right) \\
& =\frac{v-\beta_{0} T-\beta_{1}\left[\gamma_{1} k_{1}(T) C_{A}^{2}+\gamma_{2} k_{2}(T) C_{B}+\left(a_{1}+a_{2} T\right)\right]}{\beta_{1}\left(b_{1}+b_{2} T\right)}
\end{aligned}
$$

Thus, the transformed input/output system is

$$
\beta_{1} \frac{d T}{d t}+\beta_{0} T=v
$$

\section{$\underline{\text { PI controller }}$}

$$
v=K_{c}\left[T_{\mathrm{d}}(t)-T(t)\right]+\frac{K_{c}}{\tau_{l}} \int_{0}^{t}\left[T_{\mathrm{d}}(\tau)-T(\tau)\right] d \tau
$$

\section{Observer}

$$
\begin{aligned}
\hat{C}_{A}(t)= & \frac{1}{\frac{1}{\hat{C}_{A 0}}+\int_{0}^{t} k_{1}[T(\tau)] d \tau} \\
\hat{C}_{B}(t)= & \exp \left\{-\int_{0}^{t} k_{2}[T(\tau)] d \tau\right\} \\
& \int_{0}^{t}\left(\frac{k_{1}[T(\tau)]}{\left[\frac{1}{\hat{C}_{A 0}}+\int_{0}^{\tau} k_{1}[T(\xi)] d \xi\right\}^{2}} \exp \left\{\int_{0}^{\tau} k_{2}[T(\xi)] d \xi\right\}\right) d \tau
\end{aligned}
$$

Remark 11 . The process dynamics with $f, g$, and $h$ given by Eqs. 42,43 , and 44 is not linearizable in the sense of Su-HuntMeyer. It can be easily verified that the first two conditions of Eq. 10 (or, equivalently the involutivity condition in Eq. 11) imply that $d k_{1} / d T$ must be proportional to $d k_{2} / d T$, i.e., that the activation energies must be the same. Similar extremely restrictive conditions result from the involutivity condition for more complex reaction systems. So, temperature control problems for 
chemical reactors are a class of problems where the $\mathrm{Su}$-HuntMeyer approach fundamentally breaks down.

The performance of the proposed algorithm was evaluated by numerical simulations. The optimal trajectory calculated by Ray and Szekely (1973) is assumed to be reasonably approximated by

$$
T_{\mathrm{d}}(t)=54+71 \exp \left(-2.5 \times 10^{-3} t\right)
$$

The parameters and correlations were given typical values in literature (Coulson and Richardson, 1977) as follows:

$$
\begin{gathered}
\rho=1,000, \quad C_{p}=1 \\
-\Delta H_{1}=4.18 \times 10^{4}, \quad-\Delta H_{2}=8.36 \times 10^{4} \\
A_{j} / V=30 \quad A_{c} / V=17 \\
T_{s, \text { max }}=150, \quad T_{s, \text { min }}=70, \quad T_{c}=25 \\
U_{c, \text { max }}=4.42, \quad U_{c, \text { min }}=1.39, \quad U_{j}=1.16 \\
\frac{1}{U_{c}}=\frac{1}{4,550 \bar{F}_{c}^{0.8}}+\frac{1}{10.8}
\end{gathered}
$$

where $F_{c}$ denotes the coolant flow rate.

Figure 3 shows the simulated closed-loop response under the condition of perfect model and exact initialization of the concentration observer. The values of the two manipulated variables, steam temperature and coolant flow rate, are also shown. The following design parameters were used: $\beta_{0}=1, \beta_{1}=3,600, K_{c}=$ 100 , and $\tau_{I}=3,600$. It can be seen that the control is saturated at the maximum steam temperature and the minimum coolant flow rate up to $t \simeq 180$ in order to make the reactant (which is initially at $25^{\circ} \mathrm{C}$ ) reach the desired trajectory as quickly as possible. After that point, a good temperature tracking was obtained by adjustment of heating and cooling rate through manipulation of the parametric variable $u$.

The effect of process noise on the control performance was studied. Temperature measurement error was simulated by adding zero-mean random numbers with standard deviation of $\Delta T$. Other process noises were also taken into account by adding a random disturbance with standard deviation $w$ to the process dynamics, Eq. 41. However, any significant effect of these noises was not observed until the magnitude of the simulated errors became quite large. Figure 4 shows the response obtained for $\Delta T=5$ and $w=0.028$. This process noise introduces an error ranging up to $15 \%$ in the righthand side of Eq. 41 . Despite the relatively large noise, a good disturbance rejection was obtained.

The effect of modeling errors and estimation errors was also studied with the parameters $E_{1}, E_{2}, A_{10}, A_{20}, \hat{C}_{A 0}$ perturbed by a significant amount (30-100\%) in Eqs. 47 and 50 . The resulting closed-loop response was almost identical to that depicted in Figure 3. Since in our design method modeling or state estimation errors only affect the value of $u$ calculated by Eq. 47 , those errors have the same effect as a process disturbance $w$ of appropriate size. Accordingly, the robust performance observed in the simulation can be explained by the good disturbance rejection ability mentioned in the previous paragraph.

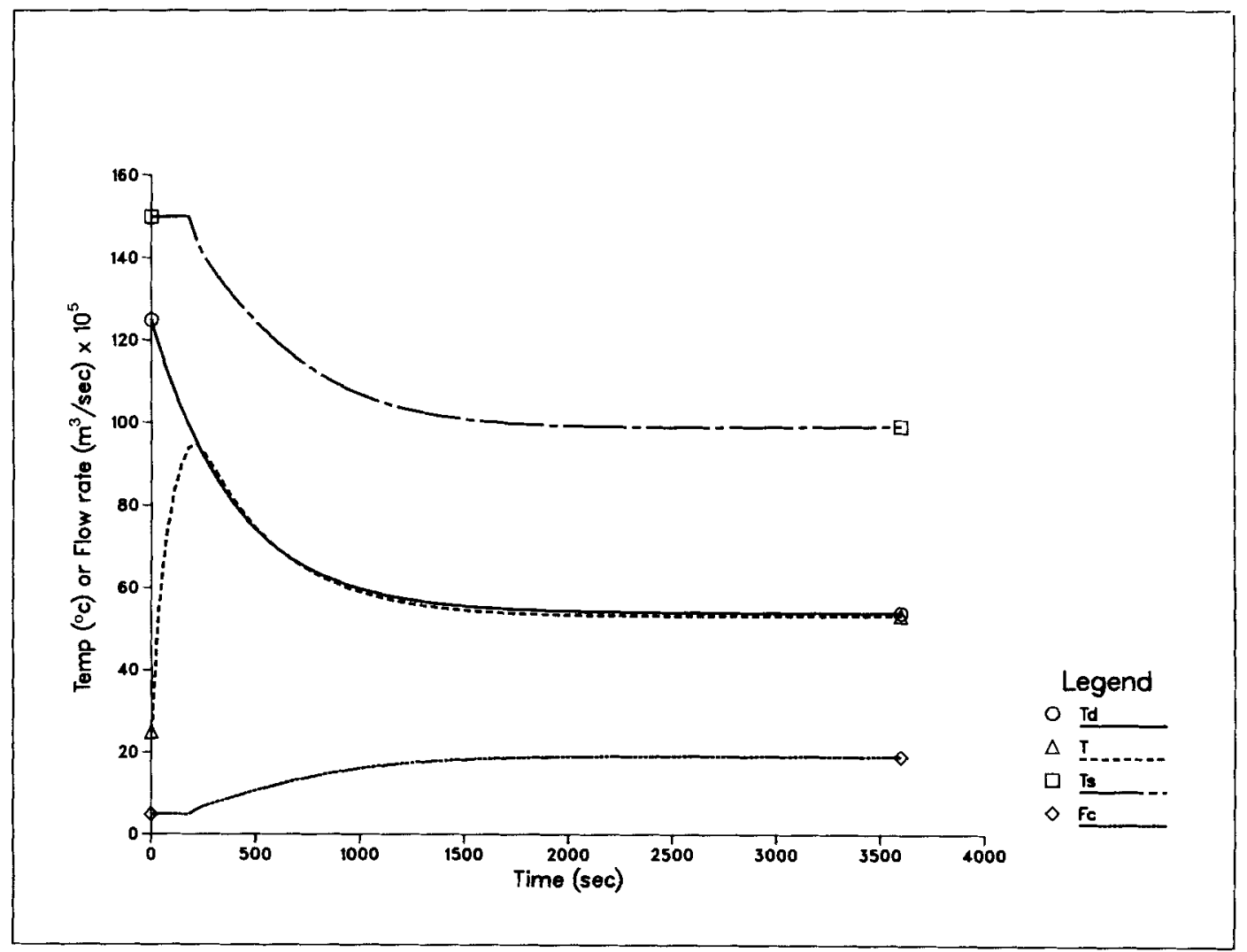

Figure 3. GLC temperature tracking without process noise. 


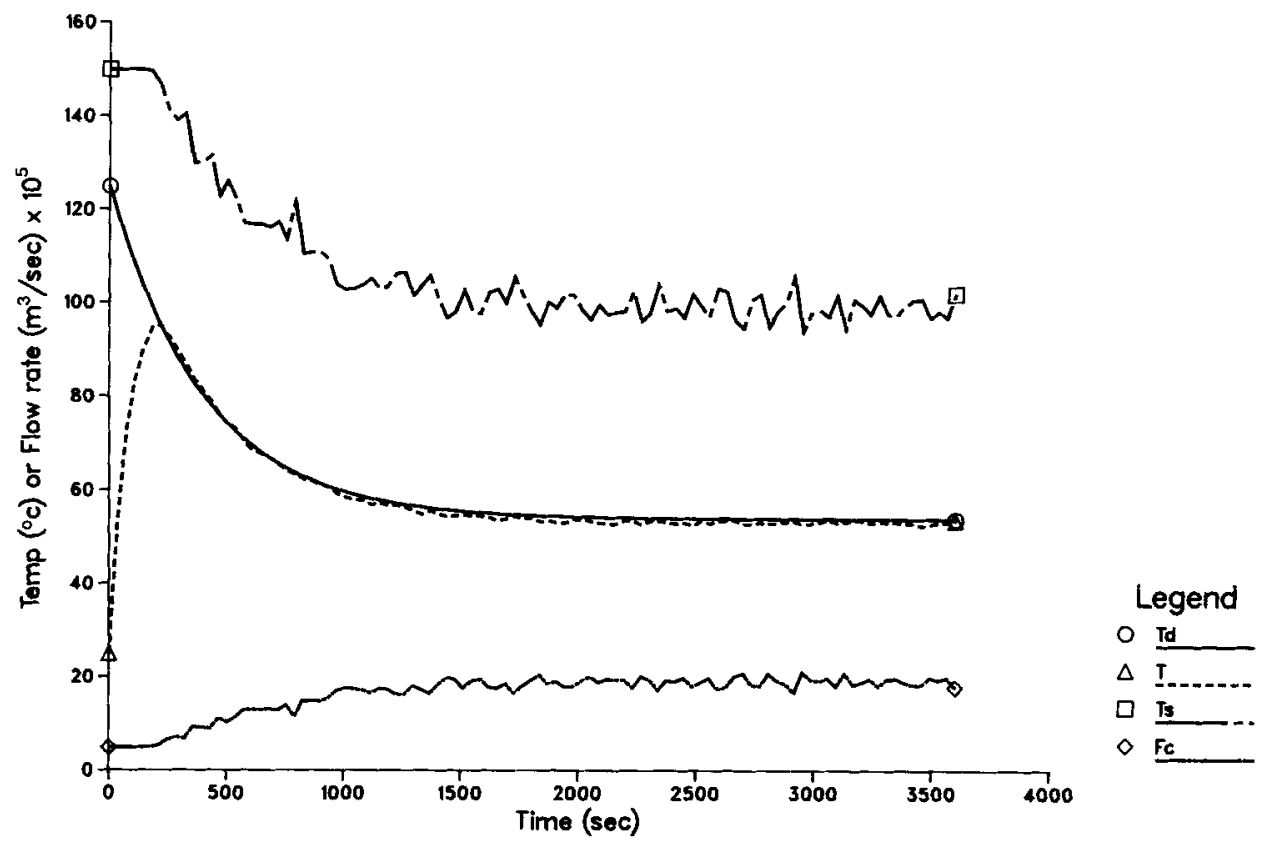

Figure 4. GLC temperature tracking with added process noise.

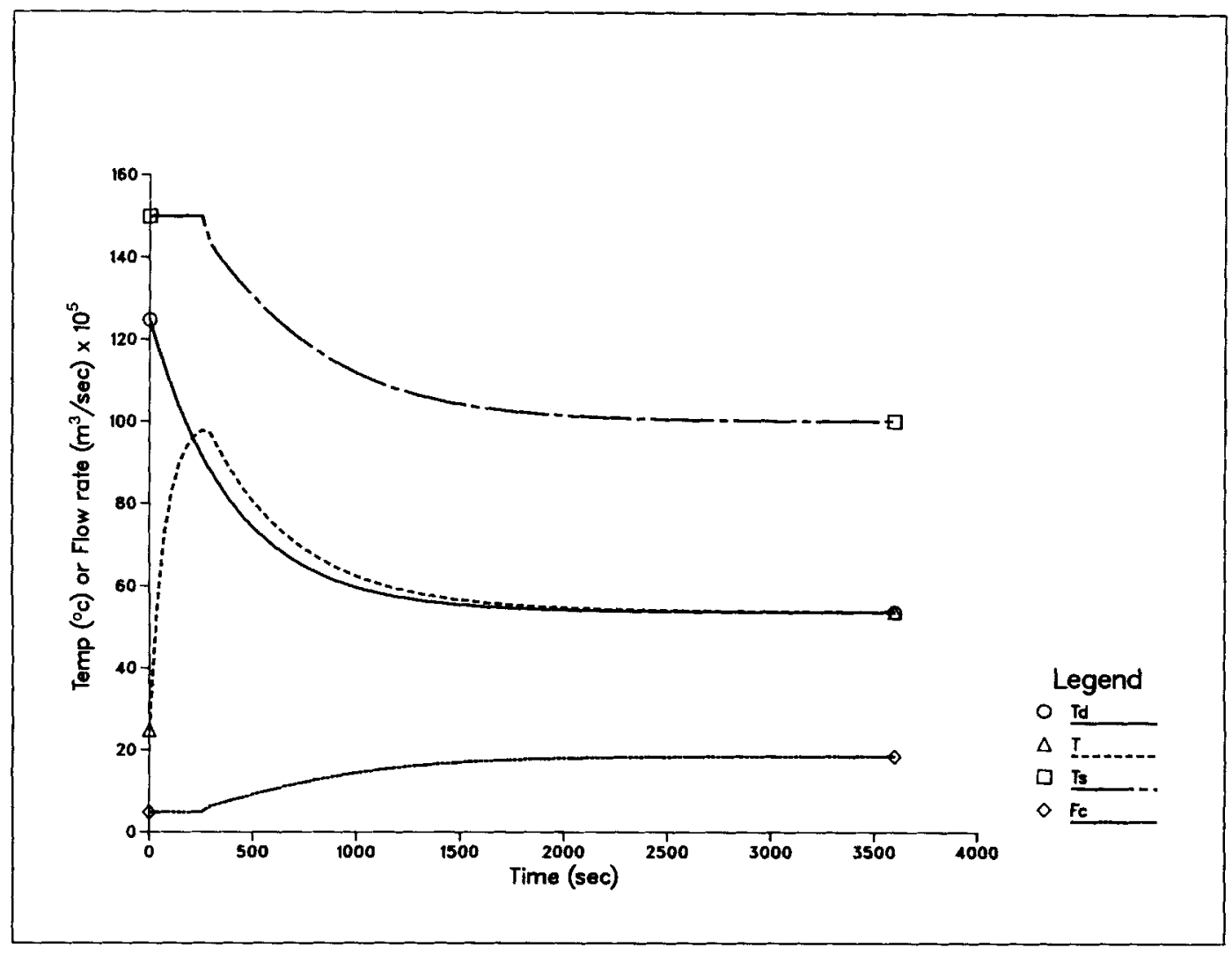

Figure 5. PI temperature tracking without process noise. 


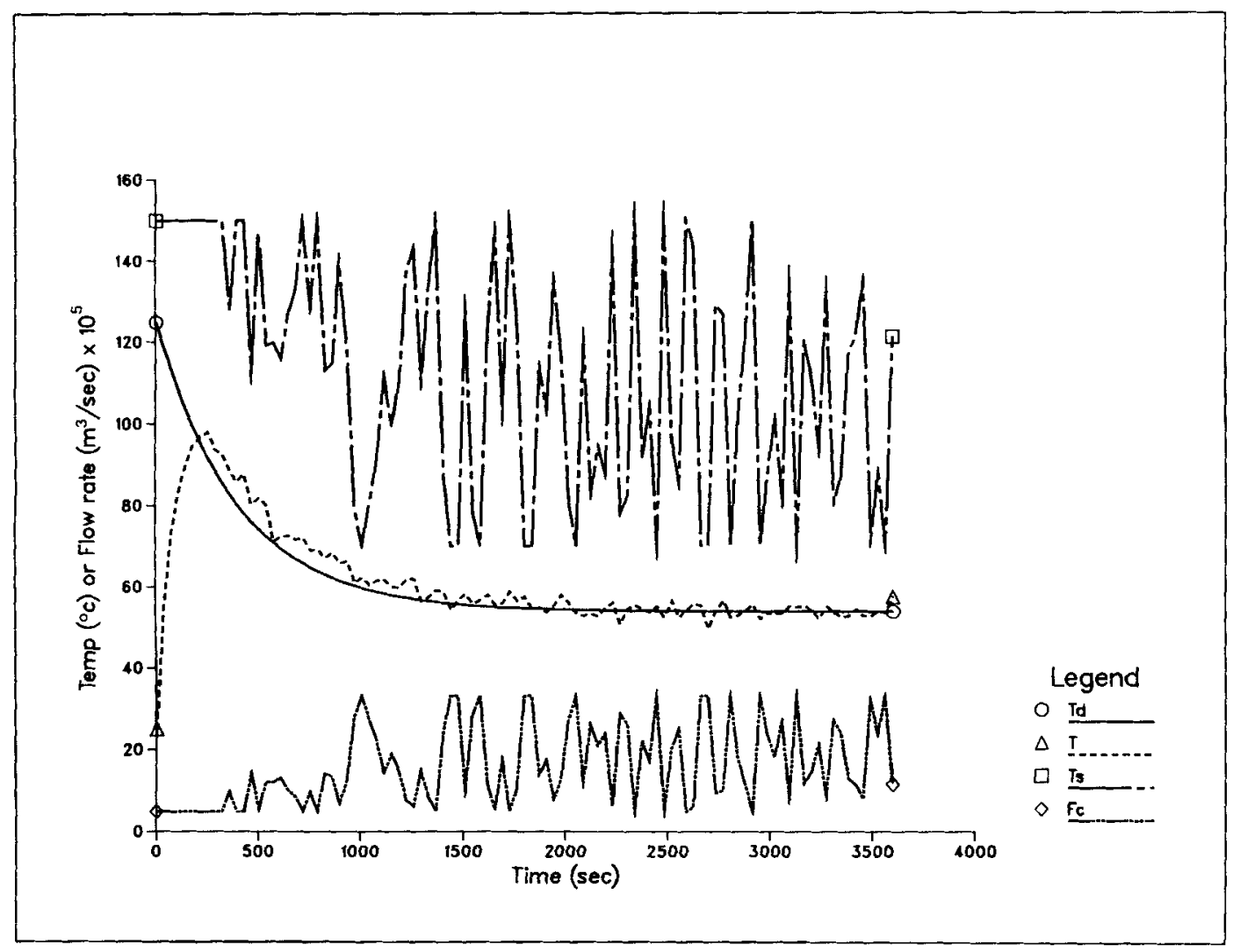

Figure 6. PI temperature tracking with added process noise.

It may be of interest to compare the performance of the GLC with that of conventional PI controllers. Of course, there is no established procedure for tuning the PI controller parameters for nonlinear tracking problems like this batch reactor; the controller tuning will have to be based on a trial-and-error procedure. This makes a good contrast to the systematic tuning procedure that is a part of the GLC synthesis. Figures 5 and 6 show the closed-loop responses obtained with a classical PI controller under the same process conditions as Figures 3 and 4, respectively, except for the values of $K_{c}=0.1, \tau_{I}=0.1$, which were determined by trial and error. Figure 5 shows a comparable performance to that in Figure 3. However, as can be seen in Figure 6 , the control action $u$ is so sensitive to small changes in $T$ that it becomes very wild under process noise.

\section{Acknowledgment}

Financial support from the Amoco Foundation is gratefully acknowledged. The authors wish to thank E. Gilbert for many useful discussions.

\section{Notation}

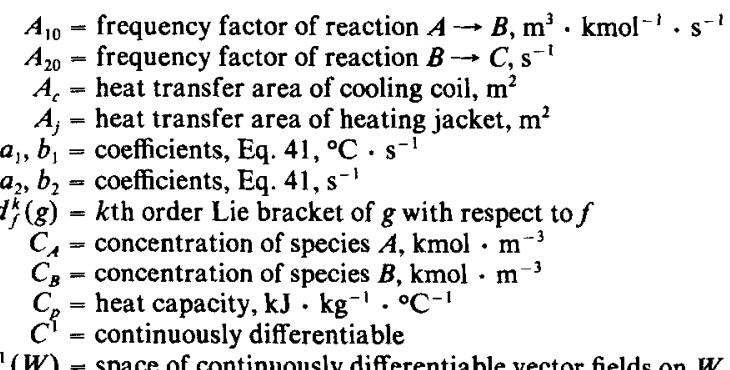

$C^{\infty}=$ infinitely many times continuously differentiable

$E$ = activation energy, $\mathrm{kJ} \cdot \mathrm{kmol}^{-1} \cdot \mathrm{K}^{-1}$

$F_{c}=$ coolant flow rate, $\mathrm{m}^{3} \cdot \mathrm{s}^{-1}$

$f, g=$ vector fields that characterize the state model

$\Delta H=$ heat of reaction, $\mathrm{kJ} \cdot \mathrm{kmol}^{-1}$

$h=$ state/output map

$K_{c}=$ proportional gain

$k_{1}=$ rate constant of reaction $A \rightarrow B, \mathrm{~m}^{3} \cdot \mathrm{kmol}^{-1} \cdot \mathrm{s}^{-1}$

$k_{2}=$ rate constant of reaction $B \rightarrow C, \mathrm{~s}^{-1}$

$L_{f}^{k}(h)=k$ th order Lie derivative of $h$ with respect to $f$

$\mathcal{L}_{r}=$ linear differential operator

$l=$ observer gain vector

$N=$ set of natural numbers

$N_{t}(x)=\epsilon$-neighborhood of $x$ in $W$

$N_{\eta}(f)=\eta$-neighborhood of $f$ in $C^{1}(W)$

$n=$ number of states

$\mathrm{R}=$ ideal gas constant, $\mathrm{kJ} \cdot \mathrm{kmol}^{-1} \cdot \mathrm{K}^{-1}$

$\boldsymbol{R}=$ set of real numbers

$r=$ linearizability index

$T=$ batch reactor temperature, ${ }^{\circ} \mathrm{C}$

$T_{c}=$ coolant temperature, ${ }^{\circ} \mathrm{C}$

$T_{\mathrm{d}}=$ desired temperature, ${ }^{\circ} \mathrm{C}$

$T_{s}=$ steam temperature, ${ }^{\circ} \mathrm{C}$

$t=$ time, s

$U_{c}=$ overall heat transfer coefficient of cooling coil, $\mathrm{kJ} \cdot \mathrm{m}^{-2}$. ${ }^{\circ} \mathrm{C}^{-1} \cdot \mathrm{s}^{-1}$

$U_{j}=$ overall heat transfer coefficient of heating jacket, $\mathrm{kJ} \cdot \mathrm{m}^{-2}$. ${ }^{\circ} \mathrm{C}^{-1} \cdot \mathrm{s}^{-1}$

$u=$ control variable

$V=$ volume, $\mathrm{m}^{3}$

$v=$ transformed control variable

$W=$ open set in state space

$w=$ mean process noise, ${ }^{\circ} \mathrm{C} \cdot \mathrm{s}^{-1}$

$x=$ state vector

$y=$ output variable

$y_{\mathrm{d}}=$ desired output

$z=$ augmented state, variable in Eqs. 27, 28 


\section{Greek letters}

$\beta=$ GLC design parameters, Eq. 15

$\gamma_{1}, \gamma_{2}=$ coefficients, Eq. $41,{ }^{\circ} \mathrm{C} \cdot \mathrm{s}^{-1}$

$\Delta T=$ mean temperature measurement noise, ${ }^{\circ} \mathrm{C}$

$x, \lambda=$ transformed vector fields

$\rho=$ density, $\mathrm{kg} \cdot \mathrm{m}^{-3}$

$\tau_{I}=$ reset time, $s$

$\Xi=$ Su-Hunt-Meyer transformation

$\xi=$ transformed state variable, Eq. 7

$\Psi=$ transformation, Eq. 21

$\boldsymbol{\Omega}=$ input-output linearizing transformation

\section{Set-theoretic symbols}

$$
\begin{aligned}
& €=\text { belongs to } \\
& \complement=\text { subset }
\end{aligned}
$$

\section{Superscripts}

$\hat{~}=$ estimated quantity

- = inexact quantity

* = equilibrium point

\section{Literature cited}

Banks, S. P., “A Note on Nonlinear Observers," Int. J. Control, 34, 185 (1981).

Bestle, D., and M. Zeitz, "Canonical Form Observer Design for Nonlinear Time-variable Systems," Int. J. Control, 38, 419 (1983).

Boothby, W. M., An Introduction to Differentiable Manifolds and Riemannian Geometry, Academic Press, New York (1975).

Brockett, R. W., "Feedback Invariants for Nonlinear Systems," Proc, 7th IF AC World Cong., Helsinki (1978).

Cluett, W. R., S. L. Shah, and D. G. Fisher, "Adaptive Control of a Batch Reactor," Chem. Eng. Commun., 38, 67 (1985).

Coulson, J. M., and J. F. Richardson, Chemical Engineering, 3rd ed., Pergamon, Elmsford, NY (1977).

Foss, A. S., "Critique of Chemical Process Control Theory," AIChE J., 19, 209 (1973).

Gilbert, E., and I. J. Ha, "An Approach to Nonlinear Feedback Control with Applications to Robotics," IEEE Trans. Syst. Man. Cybern., SMC-14, 879 (1984).

Ha, I. J., and E. Gilbert, "Robust Tracking in Nonlinear Systems and its Applications to Robotics," Proc. 24th IEEE CDC, Ft. Lauderdale, FL, 1009 (1985).

Hirsch, M. W., and S. Smale, Differential Equations, Dynamic Systems, and Linear Algebra, Academic Press, New York (1974).
Hirschorn, R. M., "Invertibility of Nonlinear Control Systems," SIAM J. Control Optim., 17, 289 (1979).

Hoo, K. A., and J. C. Kantor, "An Exothermic Continuous StirredTank Reactor Is Feedback Equivalent to a Linear System," Chem. Eng. Commun., 37, 1 (1985).

- "Linear Feedback Equivalence and Control of an Unstable Biological Reactor," Chem. Eng. Commun., 46, 385 (1986).

Hunt, L. R., R. Su, and G. Meyer, "Global Transformations of Nonlinear Systems," IEEE Trans. Auto. Control, AC-28, 24 (1983).

Jutan, A., and A. Uppal, "Combined Feedforward-Feedback Servo Control Scheme for an Exothermic Batch Reactor," Ind. Eng. Chem. Process Des. Dev., 23, 597 (1984).

Luenberger, D. G., "Observers for Multivariable Systems," IEEE Trans. Auto. Control, AC-11, 190 (1966).

Kailath, T., Linear Systems, Prentice Hall, Englewood Cliffs, NJ (1980).

Kantor, J. C., "Stability of State Feedback Transformation for Nonlinear Systems-Some Practical Considerations," Proc. 1986 Am. Control Conf., Seattle, 1014 (1986).

Kou, S. R., D. L. Elliott, and T. J. Tarn, "Exponential Observers for Nonlinear Dynamic Systems," Inform. Control, 29, 204 (1975).

Krener, A. J., and A. Isidori, "Linearization by Output Injection and Nonlinear Observers," Syst. Control Lett., 3, 47 (1983).

Marroquin, G., and W. L. Luyben, "Experimental Evaluation of Nonlinear Cascade Controllers for Batch Reactors," Ind. Eng. Chem. Fundam., 11, 552 (1972).

Ogunnaike, B. A., "Controller Design for Nonlinear Process Systems via Variable Transformations," Ind. Eng. Chem. Process Des. Dev., 25, 241 (1986).

Ray, W. H., and J. Szekely, Process Optimization, Wiley, New York (1973).

Rippin, D. W. T., "Simulation of Single and Multiproduct Batch Chemical Plants for Optimal Design and Operation," Comp. Chem. Eng., 7, 37 (1983).

Safonov, M. G., Stability and Robustness of Multivariable Feedback Systems, MIT Press (1980).

Shinskey, F. G., Process Control Systems, 2nd ed., McGraw-Hill, New York (1979).

Su, R., "On the Linear Equivalents of Nonlinear Systems," Syst. Control Lett., 2, 48 (1982).

Su, R., G. Meyer, and L. R. Hunt, "Robustness in Nonlinear Control," Differential Geometric Control Theory, R. W. Brockett, R. S. Millman, and H. J. Sussman, eds., Birkhauser, Boston, 316 (1983).

Wallman, P. H., "Reconstruction of Unmeasured Quantities for Nonlinear Dynamic Processes," Ind. Eng. Chem. Fundam., 18, 327 (1979).

Manuscript received May 6, 1986, and revision received Sept. 2, 1986. 